Special issue of the 3rd International Conference on Computational and Experimental Science and Engineering (ICCESEN 2016)

\title{
Quantitative Characteristic X-Ray Analysis for Different Compound Samples by Using Monte Carlo Method
}

\begin{abstract}
H.O. TeKin ${ }^{a, b, c, *}$, E.E. Altunsoy ${ }^{b, c}$, T. Manici ${ }^{c}$ And B. Yilmaz ${ }^{d}$
${ }^{a}$ Uskudar University, Vocational School of Health Service, Radiotherapy Department, Istanbul, Turkey

${ }^{b}$ Uskudar University, Vocational School of Health Service, Medical Imaging Department, Istanbul, Turkey

${ }^{c}$ Uskudar University, Medical Radiation Research Center (USMERA), Istanbul, Turkey

${ }^{d}$ Okan University, Faculity of Medicine, Department of Radiology, Istanbul, Turkey

X-ray spectrometry is an elemental analysis technique with broad application in science and industry. It is based on the principle that individual atoms, when excited by an external energy source emit X-ray photons of a characteristic energy or wavelength. Here we obtained the characteristic X-ray peaks of different brass compounds and compared the intensity of characteristic peaks by changing with $\mathrm{Zn}$ rate. In this study we modelled different brass samples by using Monte Carlo method and changed the zinc rate in brass samples for each compound. We obtained the characteristic X-ray peaks of different compounds and compared the intensity of characteristic peaks by changing with $\mathrm{Zn}$ rate. We achieved a good agreement between peak intensity and corresponding element rate in compound. Also we obtained that characteristic peak rate increases by corresponding element rate in compound. This study shows that Monte Carlo method is very effective method to simulate material features due to their characteristic peaks and their intensities.
\end{abstract}

DOI: 10.12693/APhysPolA.132.439

PACS/topics: Monte Carlo, characteristic X-ray, brass

\section{Introduction}

$\mathrm{X}$-rays occur when the fast electrons interact with matter. During this process, kinetic energy of electrons is basically converted into heat energy but some is also converted into electromagnetic energy in the form of photons. X-ray photons are a form of electromagnetic radiation produced after the ejection of an inner orbital electron and following transition of atomic orbital electrons from states of high to low energy. When a monochromatic beam of X-ray photons falls onto a specimen some basic phenomena may result in some effects such as absorption, scatter and fluorescence. These three phenomena forms are the basis of three important X-ray methods: the absorption technique is the basis of radiographic analysis, the scattering effect is the basis of X-ray diffraction, and the fluorescence effect is the basis of XRF spectrometry. When high energy photons (X-rays or gammarays) are absorbed by atoms, inner shell electrons are ejected from the atom, becoming photoelectrons [1]. This leaves the atom in an excited state, with a vacancy in the inner shell. Outer shell electrons then fall into the vacancy, emitting photons with energy equal to the energy difference between the two states. Since each element has a unique set of energy levels, each element emits a pattern of X-rays characteristic of the element, termed characteristic X-rays. Figure 1 shows the generation of the characteristic X-ray from atomic orbit. The intensity of the X-rays increases with the concentration of the corresponding element.

\footnotetext{
* corresponding author; e-mail: huseyinozan.tekin@uskudar.edu.tr
}

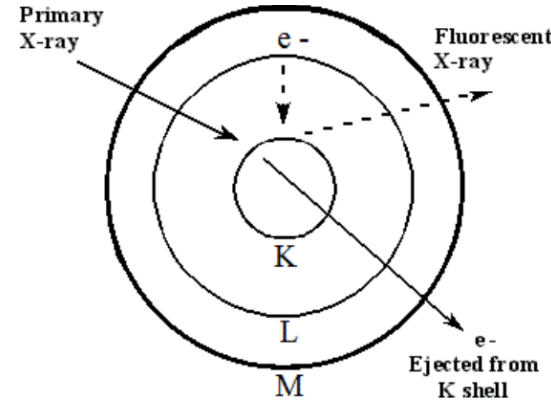

Fig. 1. Characteristic X-ray.

X-ray fluorescence is defined as the emission of characteristic secondary X-rays from a material that has been excited by bombarding with high energy X-rays or gamma rays. The phenomenon is widely used for elemental analysis. When an X-ray is absorbed by a sample atom, the absorbing atom is left in an excited state. It subsequently relaxes, emitting its own characteristic X-rays (secondary fluorescence). Since an X-ray can be absorbed only in an interaction with an electron having a binding energy less than the energy of the absorbed $\mathrm{X}$-ray, the energy of the secondary fluorescence is necessarily less than the energy of the primary X-ray [2]. Particular elements in compounds may directly estimated from diffraction line. Because of the intensity of an Xray diffraction pattern directly proportional to the concentration of the component producing it, when due allowance is made for absorption effects, it has been possible to develop methods of quantitative analysis based on diffracted intensities [3]. The diffracted intensity of any reflection from any crystalline compound can be related to the composition of the compound and its matrix, and 
to the instrumental geometry [4]. In the field of materials analysis, it is frequently used to quantitative X-ray analysis and its various methods for the different workspaces. Numerous methods have been developed to use peak intensities for quantitative analysis of diffraction data [5]. When analytical samples are irradiated with X-rays emitted from an X-ray tube or radioactive source, fluorescent $\mathrm{X}$-rays are generated in the sample and can be measured for quantitative analysis of its elements [6]. In this study we calculated the intensities of the characteristic peaks in different compounds and compared them by considering the $\mathrm{Zn}$ rate in compound. Intensities are usually measured as a rate (e.g., the number of X-ray photons detected per second) or as the total number of X-ray photons detected in a given time period (e.g., number of photons in $10 \mathrm{~s}$ counting time). These intensities are also usually normalized to the electron beam current for quantitative purposes since the number of X-ray produced is exactly proportional to the number of electron hitting the sample.

\section{Material and method}

One of the general-purpose in this study that we developed is a new Monte Carlo (MC) approach for different types of brass samples by using the WinX Ray code [6]. A screenshot of program Fig. 2 shows the structure of the MC program in below. In Fig. 2, we can see the trajectory and interaction volume of X-rays in a sample. Various simulation investigations and estimation studies on medical applications and for other aims such as computational methods are found in literature [7-19].

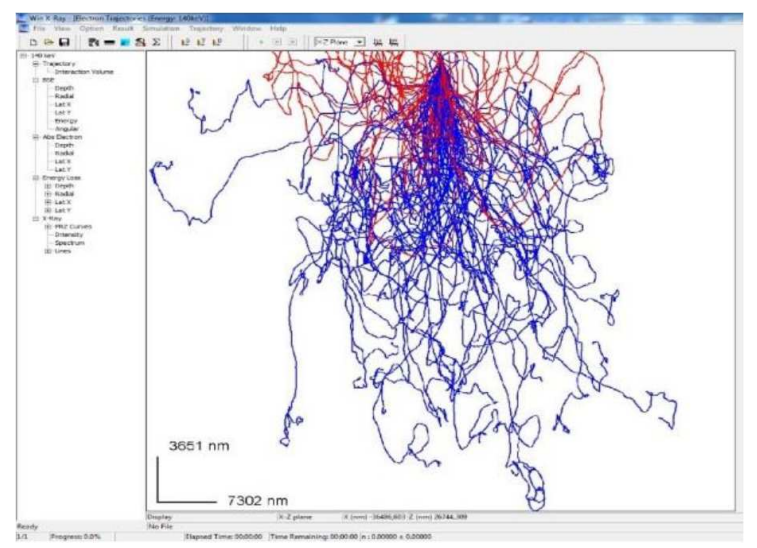

Fig. 2. Schematic structure of the MC program.

A schematic representation of modelled geometry is shown in Fig. 2. It is clear from Fig. 3 that produced $\mathrm{X}$-rays are directed onto brass sample and an energy dispersive detector is counted in the spectra of specimen.

Before the comparison studies on different compounds we simulated the pure $\mathrm{Cu}$ and $\mathrm{Zn}$ spectra and observed characteristic peaks and compared with experimental data [20]. We obtained good agreement of experimental results and Monte Carlo simulation. Figure 4 shows the characteristic $K_{\alpha 1}(8,047.78 \mathrm{keV})$ and $K_{\beta 1}(8,905.29 \mathrm{keV})$

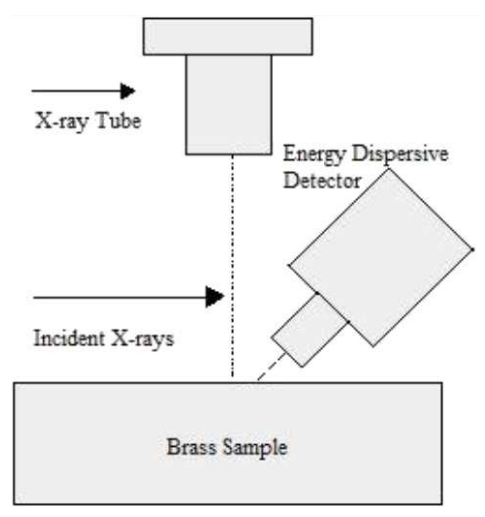

Fig. 3. Schematic view of MC.

peaks of $\mathrm{Cu}$ and Fig. 5 shows the characteristic $K_{\alpha 1}$ $(8,632.86 \mathrm{keV})$ and $K_{\beta 1}(9,572.0 \mathrm{keV})$ peaks of $\mathrm{Zn}$.

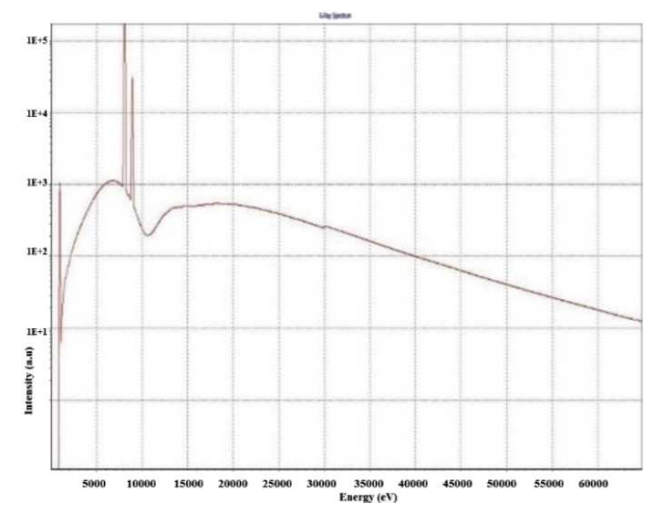

Fig. 4. $\quad K_{\alpha 1}$ and $K_{\beta 1}$ peaks of pure $\mathrm{Cu}$.

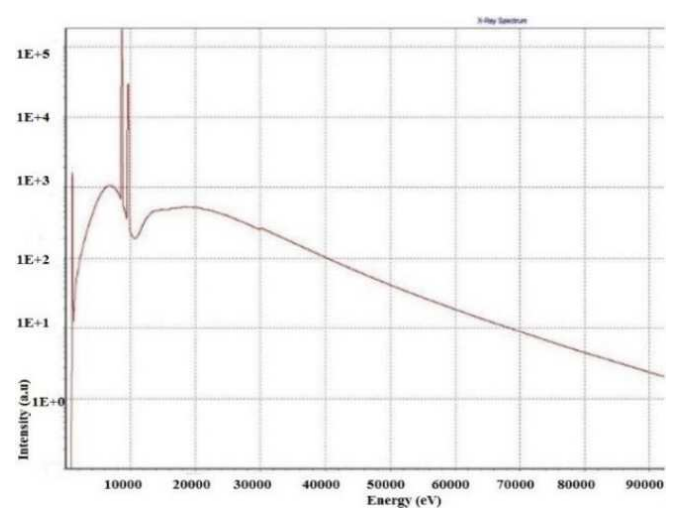

Fig. 5. $\quad K_{\alpha 1}$ and $K_{\beta 1}$ peaks of pure $\mathrm{Zn}$.

\section{Results}

X-ray analytical results of pure brass samples are illustrated and $K_{\alpha}$ and $K_{\beta}$ X-rays have been studied in some previous works [21]. In this study we consider the most common used brass samples and their $\mathrm{Cu}-\mathrm{Zn}$ ratios. Characteristic peak identification is an analytical technique used to determine the elemental composition of inorganic based materials also using for identification 
of materials. In this study we calculated each sample compound spectra and observed the $\mathrm{Cu}$ and $\mathrm{Zn}$ characteristic peaks in spectrum. For instance, Fig. 6 shows the sample ID 5 sample spectra.

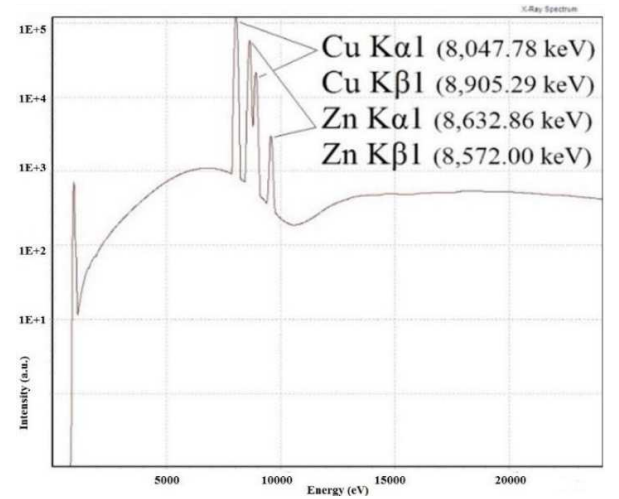

Fig. 6. $K_{\alpha 1}$ and $K_{\beta 1}$ peaks of a compound of study (sample ID 5).

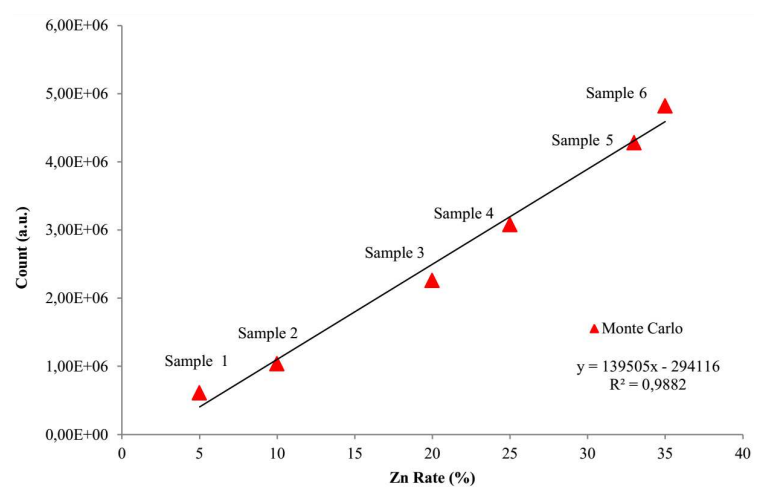

Fig. 7. Count and Zn rate in different brass compound.

Elemental rates of different brass compounds.

TABLE I

\begin{tabular}{c|c|c|c|c}
\hline \hline Sample & $\mathrm{Cu}[\%]$ & $\mathrm{Zn} \mathrm{[ \% ]}$ & $\mathrm{Zn} K_{\alpha 1}$ & $\mathrm{Zn} K_{\beta 1}$ \\
\hline 1 & 65 & 35 & $6,23 \mathrm{E}+07$ & $4,82 \mathrm{E}+06$ \\
\hline 2 & 67 & 33 & $1.72 \mathrm{E}+07$ & $4.28 \mathrm{E}+06$ \\
\hline 3 & 75 & 25 & $4.47 \mathrm{E}+06$ & $3.08 \mathrm{E}+05$ \\
\hline 4 & 80 & 20 & $1.91 \mathrm{E}+06$ & $2.26 \mathrm{E}+05$ \\
\hline 5 & 90 & 10 & $1.81 \mathrm{E}+06$ & $1.04 \mathrm{E}+05$ \\
\hline 6 & 95 & 5 & $9.015 \mathrm{E}+05$ & $6.52 \mathrm{E}+03$
\end{tabular}

For each sample we changed the $\mathrm{Zn}$ rate in compound. Table I shows the elemental amount of compounds.

We can see from Table I that intensity of $K_{\alpha 1}$ and $K_{\beta 1}$ is proportional to $\mathrm{Zn}$ rate in compound. Figure 7 shows relationship between counting amount and $\mathrm{Zn}$ rate in different brass compound. The ideal specimen for characteristic peak analysis is one in which the analysed volume of specimen is representative of the total specimen, which is, itself, representative of the sample submitted for analysis. We prepared six specimens with different elemental rates in compound. For each specimen we changed $\mathrm{Zn}$ rate by using industrial data [22].
Also we can see a linear increase from Table I between peak intensity and $\mathrm{Zn}$ rate in compound. It shows that Monte Carlo approach provided the accuracy of experimental studies.

\section{Conclusion}

As opposed to the continuous spectrum of bremsstrahlung radiation, characteristic radiation is represented by a line spectrum. As each element has a specific arrangement of electrons at discrete energy levels then it can be appreciated that the radiation produced from such interactions is characteristic of the element involved. This study shows that Monte Carlo method is very effective method to simulate material features due to their characteristic peaks and their intensities.

\section{References}

[1] R. Jenkins, in: X-Ray Techniques: Overview. Encyclopaedia of Analytical Chemistry, R.A. Meyers, 2000, p. 13269.

[2] J.I. Goldstein, D.E. Newbury, D.C. Joy, C.E. Lyman, P. Echlin, E. Lifshin, Scanning Electron Microscopy and X-Ray Microanalysis, Springer Science and Business Media, LLC, New York 2003.

[3] F.H. Chung, J. Appl. Crystallogr. 7, 519 (1974).

[4] H.P. Klug, L.E. Alexander, X-Ray Diffraction Procedures, Wiley, New York 1954.

[5] R. Jenkins, R. Snyder, Introduction to X-Ray Powder Diffractometry. Chemical Analysis, Wiley, New York 1996.

[6] Tomoya Arai, The Rigaku J. 21, 26 (2004).

[7] H.O. Tekin, Sci. Technol. Nucl. Install. 2016 , 6547318 (2016).

[8] I. Akkurt, H.O. Tekin, A. Mesbahi, Acta Phys. Pol. A 128, B- (332)2015.

[9] H.O. Tekin, U. Kara, J. Commun. Comput. 13, 32 (2016).

[10] H.O. Tekin, T. Manici, C. Ekmekci, J. Health Sci. 4 , 131 (2016).

[11] H.O. Tekin, V.P. Singh, T. Manici, Appl. Radiat. Isot. 121, 122 (2017).

[12] N. Zeng, Z. Wang, H. Zhang, F.E. Alsaadi, Cogn. Comput. 8, 143 (2016).

[13] J. Liang, S.Y. Yuen, Cogn. Comput. 8, 693 (2016).

[14] Z. Tang, J. Lu, P. Wang, Cogn. Comput. 7, 731 (2015).

[15] M. Gunay, Acta Phys. Pol. A 128, B-113 (2015).

[16] U. Kara, A. Mesbahi, I. Akkurt, Acta Phys. Pol. A 128, B-378 (2015).

[17] N. Demir, A. Aydin, Acta Phys. Pol. A 128, B-466 (2015).

[18] M. Gunay, V.H. Sanchez Espinoza, A. Travleev, Acta Phys. Pol. A 128, B-110 (2015).

[19] İ. Akkurt, H.O. Tekin, A. Mesbahi, Acta Phys. Pol. A 128, B-332 (2015).

[20] Lawrence Berkeley National Laboratory, X-Ray Data Booklet, Access Date: 25.12.2015.

[21] European Copper Institute Publications, Types of Brass, Pub. 117, Section 6, Access Date: 27.12.2015.

[22] H.O. Tekin, U. Kara, in: RAD4 Conf. Proc. Book 1, 133 (2016). 